\title{
COMMISSIONING AND FUTURE PLANS FOR POLARIZED PROTONS IN RHIC**
}

\author{
W.W. MacKay ${ }^{\dagger}$, L. Ahrens, M. Bai, G. Bunce, E. Courant, A. Deshpande, A. Drees, W. Fischer, \\ H. Huang, K. Kurita, A.U. Luccio, Y. Makdisi, F. Pilat, V. Ptitsin, T. Roser, N. Saito, T. Satogata, \\ S. Tepikian, D. Trbojevic, N. Tsoupas, J. van Zeijts, BNL, USA, \\ H. Spinka, D. Underwood, ANL, USA, A. Lehrach, IKP, Juelich, Germany, I. Alekseev, \\ V. Kanavets, D. Svirida, ITEP, Russia, K. Imai,, J. Tojo, Kyoto Univ., Japan, \\ B. Lozowski, V. Ranjbar, Indiana Univ., USA, D. Fields, Univ. of New Mexico, USA
}

\begin{abstract}
Polarized protons were injected and accelerated in the clockwise ring of RHIC to commission the first full helical Siberian snake ever used in an accelerator. With the snake turned on, the stable spin direction is in the horizontal plane. Vertically polarized protons were injected with the snake off. The snake was adiabatically ramped to give a spin rotation of $180^{\circ}$ around a horizontal rotation axis about $13^{\circ}$ from the longitudinal. When the beam was accelerated from injection $G \gamma=46.5$ to $G \gamma=48$ the spin flipped sign as expected and polarization was preserved. At $G \gamma=48$ without the snake, no polarization was observed since several spin resonances were crossed. Eventually polarized beam was accelerated to $G \gamma=55.7(29.1 \mathrm{GeV})$. In the next proton running period we plan to run with two full helical snakes in each ring and collide both transversely and longitudinally polarized protons at an energy around $100 \mathrm{GeV}$ per beam.
\end{abstract}

\section{INTRODUCTION}

In addition to colliding heavy ions, the Relativistic Heavy Ion Collider is being fitted[1] with Siberian snakes and spin rotators for high energy collisions of polarized protons at center-of-mass energies up to $\sqrt{s}=500 \mathrm{GeV}$. Fig. 1 shows a layout of hardware for injecting and colliding polarized protons. Polarized protons have been a part of the AGS fixed target program for many years, and a new optically pumped polarized proton source (OPPIS)[2] has been added to increase the current for RHIC injection. The final RHIC configuration for polarized protons is also shown in Fig. 1. To date, the four snakes and two proton carbon Coulomb nuclear interference $(p C \mathrm{CNI})$ polarimeters have been installed, and the eight spin rotators will be installed next summer.

\subsection{Helical Siberian Snakes}

The precession of spins in electromagnetic fields is described by the Thomas-BMT equation. For transverse guide fields in an accelerator, the spin precesses $G \gamma$ faster

\footnotetext{
* Work supported in part by RIKEN and the U.S. Department of Energy.

†waldo@bnl.gov
}

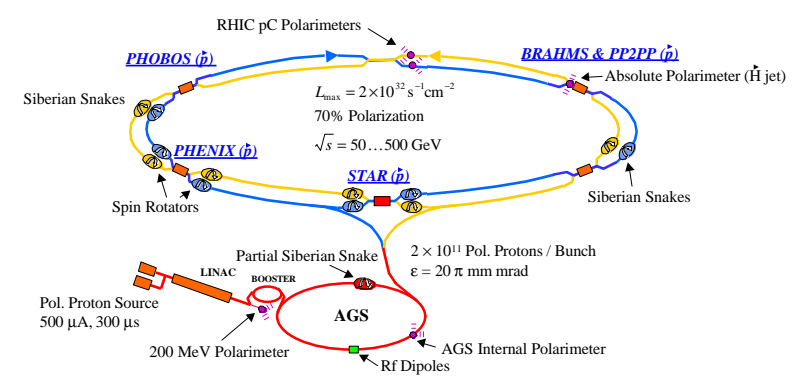

Figure 1: Layout of RHIC and injectors for polarized protons. In each of the two RHIC rings (Blue: $\mathrm{CW}$, and Yellow: $\mathrm{CCW}$ ) there will be two helical Siberian snakes and four helical spin rotators placed on either side of two interaction regions. There is a $p C \mathrm{CNI}$ polarimeter in each ring. A polarized hydrogen jet polarimeter is being built and will be installed in one of the interaction regions which is common to both rings

than the orbital direction where $G=1.7928$ is the anomalous factor $\frac{g-2}{2}$ for the proton. For a flat accelerator with only horizontal bends this gives a spin tune $\nu_{s}=G \gamma$. This means that an integer depolarizing resonance is crossed for every $523 \mathrm{MeV}$ of acceleration. These integer imperfection resonances are driven by imperfections in the lattice such as misalignments of magnets. From injection at $24.3 \mathrm{GeV}$ to top energy at $250 \mathrm{GeV}$ the spin tune would cross about 430 imperfection resonances. In addition to the imperfection resonances vertical betatron oscillations drive other depolarizing resonances called intrinsic resonances.

In each RHIC ring two snakes are placed on opposite sides and are adjusted to flip the spin exactly $180^{\circ}$ (angle $\mu$ ) about some axis in the horizontal plane. In a given snake $\phi$ is the angle between the axis of rotation and direction of the beam. The spin will be up in one half of the ring and down in the other. Provided that the two rotation axes are perpendicular (e.g., $\pm 45^{\circ}$ ) then the spin tune will be $\frac{1}{2}$. For the most part nasty things which happen to the spin in one half of the ring get undone (unwound) in the other half. If the betatron tunes are kept away from the half-integer and the orbit is sufficiently flattened then the polarization will be preserved during acceleration. Ref. [5] gives a calculation of the spin resonances in RHIC. 

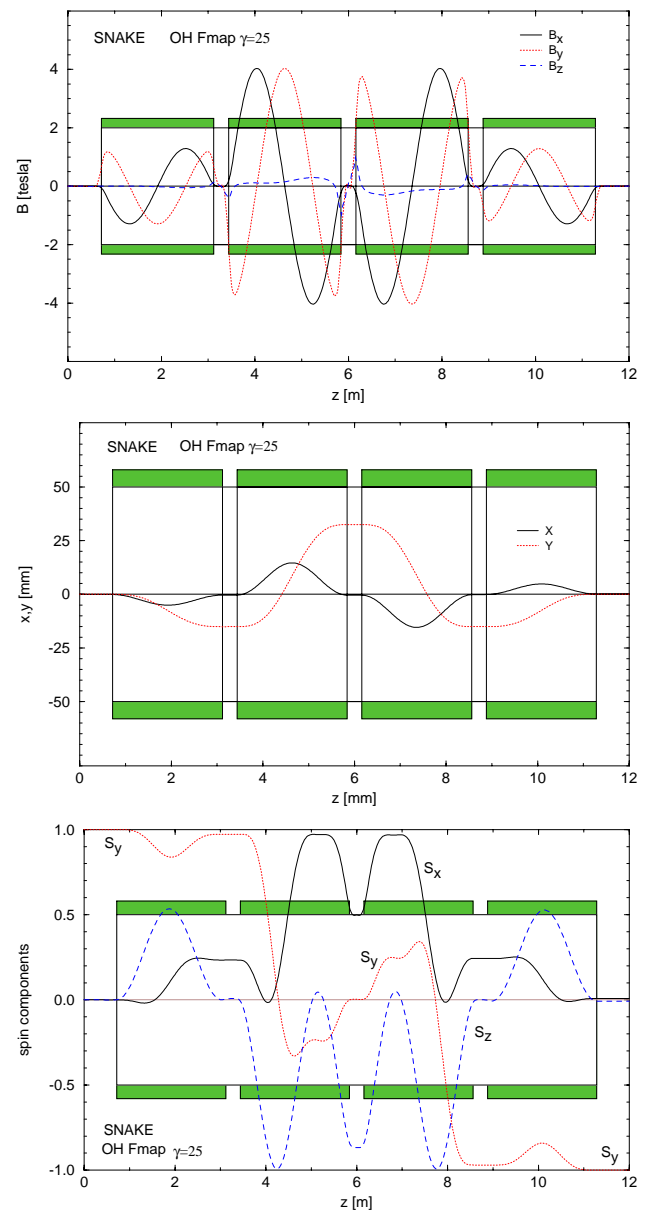

Figure 2: Nominal field, orbit and spin plots through a snake. The top plot shows the magnetic field components of the helical dipoles along the design trajectory. The middle plot shows the design trajectory through the snake. At $250 \mathrm{GeV}$ the displacement is reduced by a factor of 10 . The bottom plot shows how the spin precesses from up to down for a proton traveling through the snake.

For RHIC we chose a compact snake design with four superconducting helical dipoles that fits within the typical straight section of a dispersion suppressor half cell at the end of an arc. Each of the helices is about $2.4 \mathrm{~m}$ long with a complete right-handed $360^{\circ}$ rotation of the dipole field as shown in Fig. 2. A typical trajectory with spin rotation is also shown. Note that the spin precession is essentially independent of energy since these snakes use a transverse field. The helices are powered by two power supplies: one for the outer pair of helices, and one for the inner pair. The circuits are wired so that the polarity of the fields at the end of the helices are,,,+-+- . Fig. 3 shows how the spin rotation of a snake changes with field settings.

For the rotators we alternate right- and left-handed helices. In RHIC due to space limitations, the rotators had to be placed in straight sections which are not quite parallel to the interaction region; therefore, the required setting is energy dependent since there is additional precession in the

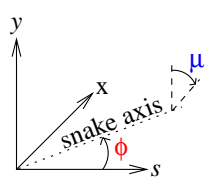

The rotation axis of the snake is $\phi$, and $\mu$ is the rotation angle.

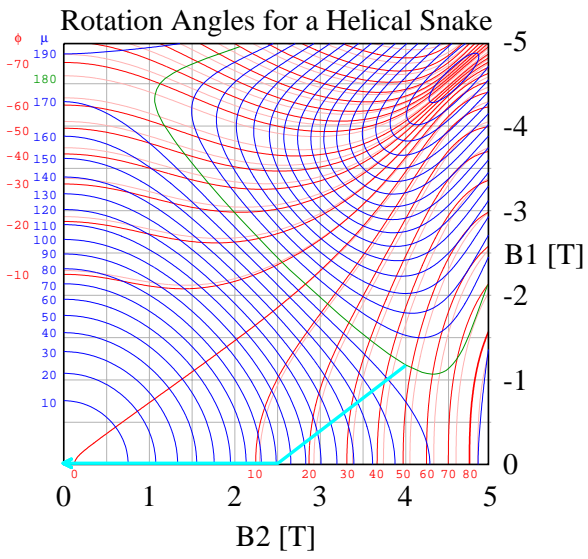

Figure 3: Contours of spin rotation for various values of the magnetic fields. Note that the $\phi$ contours shift slightly from injection (red) at $25 \mathrm{GeV}$ to storage (pink-light) at $250 \mathrm{GeV}$.

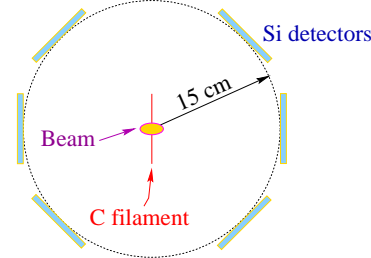

Beam's-eye view of detectors

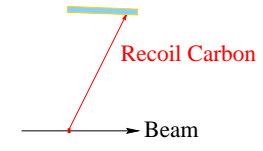

Side View
Figure 4: Layout of the polarimeter. The two horizontal silicon detectors were not installed for the initial commissioning.

horizontal plane from the IR dipoles. Because of this the rotators will only turned on after reaching storage energy.

\subsection{Polarimeters}

The $p C$ CNI polarimeter uses the spin dependence of proton-carbon in the Coulomb nuclear interference region. For vertical polarization the left-right asymmetry or recoiling carbon nuclei is measured. The polarimeter consists of silicon detectors placed inside the beam pipe. There are six detectors placed as shown in Fig. 4. The four detectors at $\pm 45^{\circ}$ allow the measurement of both vertical and radial components of polarization. Ref. [6] gives a more complete discussion of this polarimeter.

\section{COMMISSIONING THE BLUE RING}

Last fall, in the first commissioning run with polarized protons, we had only one snake and one polarimeter installed in the Blue ring. Six bunches of protons with al-

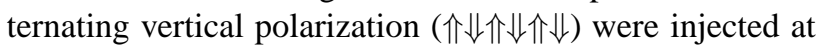
$G \gamma=46.5$ with the snake turned off. A plot of the reconstructed mass of recoils particles in the silicon detectors shown in the upper left of Fig. 5 shows that the detectors can resolve the low energy $C^{12}$ and $H e^{4}$ nuclei. The measured asymmetry (top right of Fig. 5) corresponds 
to about a $20 \%$ vertical polarization. The snake was then turned on slowly by ramping the inner and outer fields linearly to $B_{1}=-B_{2} \simeq 2.45 \mathrm{~T}$ for a $180^{\circ}$ spin rotation with $\phi \sim-13^{\circ}$. In the third plot (Fig. 5) we see the polarization has rotated into the horizontal plane. With one snake the polarization on the opposite side of the ring (where the other snake should be) the stable polarization direction is parallel to the snake's rotation axis. Since the polarimeter is about a quarter of the way or so around the ring, the stable direction at the polarimeter will vary with energy. At injection this is about $65^{\circ}$ from the longitudinal, so that the measured radial polarization should be $\sin 65^{\circ}=0.91$ of the total horizontal polarization. We then started to accelerate the beam slowly in steps. At $G \gamma$ of 48 , the polarization had rotated to radially inward. Polarization was preserved up to $G \gamma=55.7$, but lost before reaching 60.3. There is a stronger intrinsic resonance at $G \gamma \sim 58$ which most likely caused the depolarization. During commissioning the vertical orbit was corrected to no better than $\sigma_{y} \sim 0.5 \mathrm{~mm}$. For future running we will be using two snakes per ring to accelerate as well as doing a better job of flattening the orbit.

Orbit studies were done at injection with the nominal setting of the snake, $\phi=45^{\circ}$. With the higher field near $4 \mathrm{~T}$ in the middle two helices we found some distortion of the orbit which required a $0.18 \mathrm{mrad}$ correction by the vertical steering magnet just upstream of the snake. For correct spin precession the snake only needs to vary with $\beta$, not $\gamma$ so the amount of correction needed will drop as $\gamma^{-1}$.

\section{FUTURE PLANS}

Our next run with polarized protons this fall will have all four snakes and both $p C C N I$ polarimeters, but no rotators. We hope to collide polarized protons at $\sqrt{s}=200 \mathrm{GeV}$ $(G \gamma=192)$ with $50 \%$ longitudinal polarization at all six IP's. To do this we will accelerate with both snakes and then turn off one snake to rotate the spin into the horizontal plane. With $G \gamma$ being a multiple of 6 , there will be an integral number of precessions of the spin between IR's, but still $\nu_{s}=\frac{1}{2}$ with the remaining snake. At this energy the polarization at the IR's will be longitudinal if the remaining snake is adjusted to $\phi=53^{\circ}$. As the snake is turned off the spin tune deviates from 0.5 by as much as 0.13 and then back again to 0.5 . This effect can be halved by slightly changing the the $\phi$ and $\mu$ of the remaining snake as the first one is ramped down.

In the spring and summer of 2002, we will install the eight rotators, and in the following run will collide protons at $\sqrt{s}=500 \mathrm{GeV}$ with longitudinal polarization at STAR and PHENIX.

The polarized jet should be completed for installation sometime by the end of 2003 .

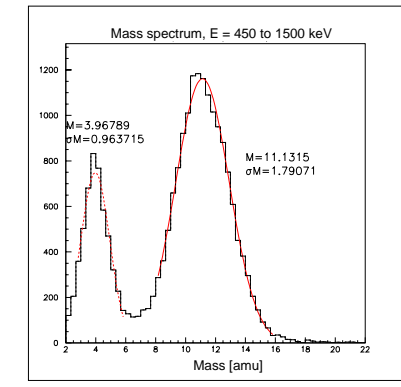

Inject, snake off
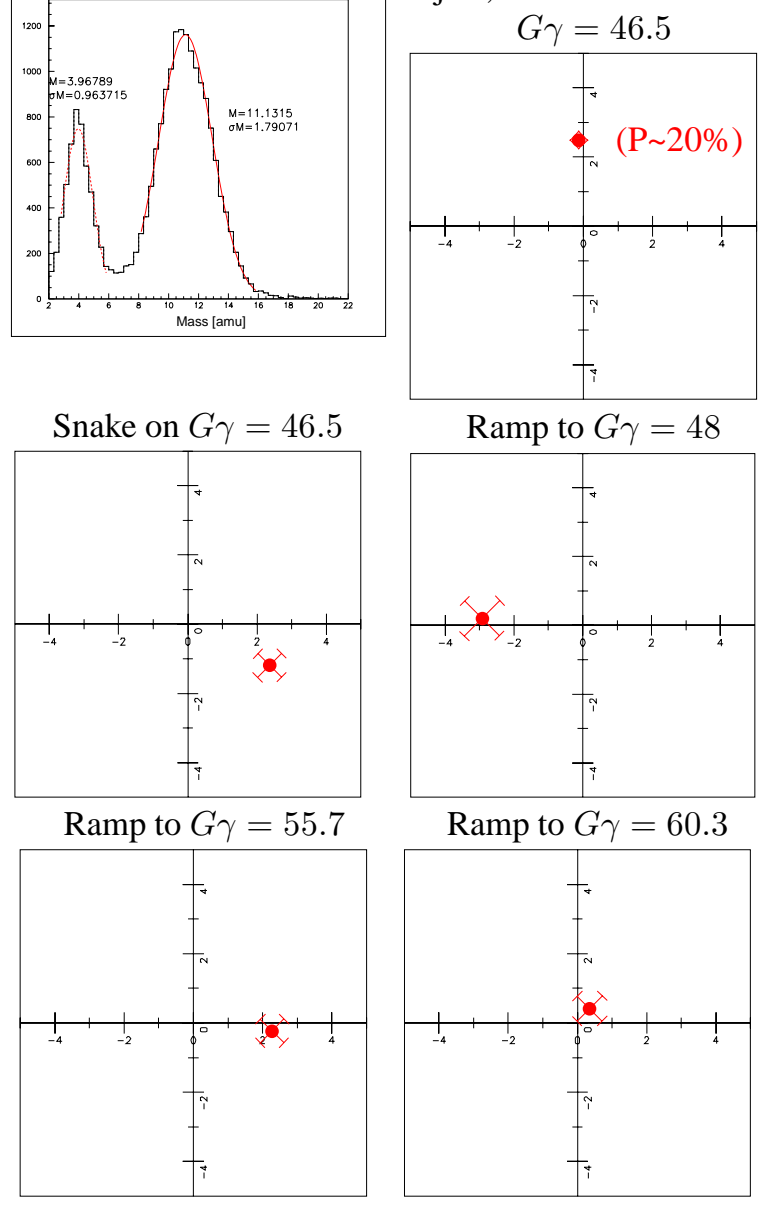

Figure 5: Polarization measurements for various conditions. The upper left figure shows a mass plot of the recoil particles from time-of-flight and energy-loss measurements. Vertically polarized protons were injected with the snake off. The snake was slowly turned on to rotate the spin into the horizontal plane with the stable spin direction about $65^{\circ}$ from the longitudinal. As the beam was accelerated the stable spin direction at the polarimeter precessed from radially outward (46.5) to inward (48). Polarization was maintained up to $G \gamma=55.7$, but was depolarized just below $G \gamma=60.3$ after crossing an intrinsic resonance.

\section{REFERENCES}

[1] I. Alekseev et al., "Polarized Proton Collider at RHIC", NIM to be published.

[2] A.N. Zelenski et al., PAC 1999, p. 108 (1999).

[3] W.W. MacKay et al., DESY-PROC-1999-03, p. 163 (1999).

[4] V. Ranjbar et al., "Fitting Helical Snake and Rotator Field Strength Measurements in RHIC", these proceedings.

[5] V. Ranjbar et al., "Mapping out the Full Spin Resonance Structure of RHIC", these proceedings.

[6] H. Huang et al., "A New Relative Proton Polarimeter for RHIC", these proceedings. 